\title{
BMJ Open Efficacy of low-level laser therapy on pain and disability in knee osteoarthritis: systematic review and meta-analysis of randomised placebo-controlled trials
}

\author{
Martin Bjørn Stausholm (D) , ${ }^{1}$ Ingvill Fjell Naterstad, ${ }^{1}$ Jon Joensen, ${ }^{1}$ \\ Rodrigo Álvaro Brandão Lopes-Martins, ${ }^{2}$ Humaira Sæbø, ${ }^{1}$ Hans Lund, ${ }^{3}$ \\ Kjartan Vibe Fersum, ${ }^{1}$ Jan Magnus Bjordal ${ }^{1}$
}

To cite: Stausholm MB, Naterstad IF, Joensen J, et al. Efficacy of low-level laser therapy on pain and disability in knee osteoarthritis: systematic review and meta-analysis of randomised placebocontrolled trials. BMJ Open 2019;9:e031142. doi:10.1136/ bmjopen-2019-031142

- Prepublication history and additional material for this paper are available online. To view these files, please visit the journal online (http://dx.doi org/10.1136/bmjopen-2019031142).

Received 18 April 2019 Revised 11 September 2019 Accepted 17 September 2019

\section{Check for updates}

C Author(s) (or their employer(s)) 2019. Re-use permitted under CC BY-NC. No commercial re-use. See rights and permissions. Published by BMJ.

${ }^{1}$ Department of Global Public Health and Primary Care, University of Bergen, Bergen, Norway

${ }^{2}$ Instituto de Pesquisa \& Desenvolvimento, Universidade do Vale do Paraíba, São José dos Campos, Brazil

${ }^{3}$ Centre for Evidence-Based Practice, Hogskulen pa Vestlandet, Bergen, Norway

Correspondence to Martin Bjørn Stausholm; m.b.stausholm@gmail.com

\section{ABSTRACT}

Objectives Low-level laser therapy (LLLT) is not recommended in major knee osteoarthritis (KOA) treatment guidelines. We investigated whether a LLLT dose-response relationship exists in KOA.

Design Systematic review and meta-analysis.

Data sources Eligible articles were identified through PubMed, Embase, Cumulative Index to Nursing and Allied Health Literature, Physiotherapy Evidence Database and Cochrane Central Register of Controlled Trials on 18 February 2019, reference lists, a book, citations and experts in the field.

Eligibility criteria for selecting studies We solely included randomised placebo-controlled trials involving participants with KOA according to the American College of Rheumatology and/or Kellgren/Lawrence criteria, in which LLLT was applied to participants' knee(s). There were no language restrictions.

Data extraction and synthesis The included trials were synthesised with random effects meta-analyses and subgrouped by dose using the World Association for Laser Therapy treatment recommendations. Cochrane's risk-ofbias tool was used.

Results 22 trials ( $n=1063)$ were meta-analysed. Risk of bias was insignificant. Overall, pain was significantly reduced by LLLT compared with placebo at the end of therapy (14.23 mm Visual Analogue Scale (VAS; 95\% Cl 7.31 to 21.14)) and during follow-ups 1-12 weeks later (15.92 mm VAS (95\% Cl 6.47 to 25.37)). The subgroup analysis revealed that pain was significantly reduced by the recommended LLLT doses compared with placebo at the end of therapy ( $18.71 \mathrm{~mm}$ (95\% Cl 9.42 to 27.99$)$ ) and during follow-ups 2-12 weeks after the end of therapy (23.23 mm VAS (95\% Cl 10.60 to 35.86$))$. The pain reduction from the recommended LLLT doses peaked during follow-ups 2-4 weeks after the end of therapy (31.87 mm VAS significantly beyond placebo (95\% Cl 18.18 to 45.56$)$ ). Disability was also statistically significantly reduced by LLLT. No adverse events were reported.

Conclusion LLLT reduces pain and disability in KOA at 4-8 J with 785-860 nm wavelength and at 1-3 J with $904 \mathrm{~nm}$ wavelength per treatment spot. PROSPERO registration number CRD42016035587.
Strengths and limitations of this study

- The review was conducted in conformance with a detailed a priori published protocol, which included, for example, laser dose subgroup criteria.

- No language restrictions were applied; four (18\%) of the included trials were reported in non-English language.

- A series of meta-analyses were conducted to estimate the effect of low-level laser therapy on pain over time.

- Three persons each independently extracted the outcome data from the included trial articles to ensure high reproducibility of the meta-analyses.

- The review lacks quality-of-life analyses, a detailed disability time-effect analysis and direct comparisons between low-level laser therapy and other interventions.

\section{INTRODUCTION}

Approximately $13 \%$ of women and $10 \%$ of men in the population aged $\geq 60$ years suffer from knee osteoarthritis (KOA) in the USA. ${ }^{1}$ $\mathrm{KOA}$ is a degenerative inflammatory disease affecting the entire joint and is characterised by progressive loss of cartilage and associated with pain, disability and reduced quality of life (QoL). ${ }^{1}$ Increased inflammatory activity is associated with higher pain intensity and more rapid KOA disease progression. ${ }^{12}$

Some of the conservative intervention options for KOA are exercise therapy, non-steroidal anti-inflammatory drugs (NSAIDs) and anti-inflammatory low-level laser therapy (LLLT). There is evidence that exercise therapy reduces pain and disability and improves QoL in persons with KOA. ${ }^{3}$ NSAIDs are recommended in most KOA clinical treatment guidelines and is probably the most frequently prescribed therapy category for osteoarthritis, despite intake of these 
drugs is associated with negative side effects, ${ }^{5}$ which is problematic, especially since the disease requires longterm treatment. Furthermore, a recently published network meta-analysis indicates that the pain relieving effect of NSAIDs in KOA beyond placebo is small to moderate (depending on drug type) ${ }^{6}$ Likewise, in the first systematic review on this topic, the pain relieving effect of NSAIDs was estimated to be only $10.1 \mathrm{~mm}$ on the $0-100 \mathrm{~mm}$ Visual Analogue Scale (VAS) better than placebo. ${ }^{7}$

LLLT is a non-invasive treatment modality, ${ }^{89}$ which has been reported to induce anti-inflammatory effects. ${ }^{9-14}$ LLLT was compared with NSAID in rats with KOA by Tomazoni et al in a laboratory; NSAID (10 mg diclofenac/ knee/session) and LLLT (830 nm wavelength, $6 \mathrm{~J} / \mathrm{knee} /$ session) reduced similar levels of inflammatory cells and metalloproteinase (MP-3 and MP-13). In addition, LLLT reduced the expression of proinflammatory cytokines (interleukin-1 $\beta$ (IL-1 $\beta$ ) and IL-6 and tumour necrosis factor $\alpha$ ), myeloperoxidase and prostaglandin $\mathrm{E}_{2}$ significantly more than NSAID did. ${ }^{1011}$

LLLT has been applied to rabbits with KOA three times per week for 8 weeks in a placebo-controlled experiment by Wang et al. ${ }^{12}$ At the end of treatment week 6, they found that LLLT had significantly reduced pain and synovitis and the production of IL-1 $\beta$, inducible nitric oxide synthase and MP-3 and slowed down loss of metallopeptidase inhibitor 1 . Two weeks later, LLLT had significantly reduced MP-1 and MP-13 and slowed down loss of collagen II, aggrecan and transforming growth factor beta, and the previous changes were sustained. ${ }^{12}$ These findings indicate that the effects of LLLT increase over time.

Pallotta et $a l^{14}$ conducted a study on LLLT in rats with acute knee inflammation, which demonstrated that even though LLLT $(810 \mathrm{~nm})$ significantly enhanced cyclooxygenase (COX-1 and COX-2) expression it significantly reduced several other inflammatory makers, that is, leucocyte infiltration, myeloperoxidase, IL-1 and IL-6 and especially prostaglandin $\mathrm{E}_{2}$. Pallotta $e t a l^{4}$ hypothesised that the increase in COX levels by LLLT was involved in a production of inflammatory mediators related to the resolution of the inflammatory process.

LLLT is not recommended in major osteoarthritis treatment guidelines. LLLT for KOA was mentioned in the European League Against Rheumatism osteoarthritis guidelines (2018) but not recommended, ${ }^{15}$ and in the Osteoarthritis Research Society International guidelines (2018), it was stressed that LLLT should not be considered a core intervention in the management of KOA. ${ }^{16}$

This may be partly due to conflicting results of two recently published systematic reviews on the current topic. ${ }^{8} 17$ The conflicting results may arise from omission of relevant trials ${ }^{817-23}$ and unresolved LLLT dose-related issues. Only Huang et $a l^{17}$ conducted a LLLT dose-response relationship investigation in KOA, that is, by subgrouping the trials by laser dose, but they did not consider that World Association for Laser Therapy
(WALT) recommends applying four times the laser dose with continuous irradiation compared to superpulsed irradiation. ${ }^{22}{ }^{24-26}$ Thus, it was unknown whether LLLT is effective in KOA, and we saw a need for a new systematic review.

The objectives of the current review were to estimate the effectiveness of LLLT in KOA regarding knee pain, disability and QoL, and we only considered placebo-controlled randomised clinical trials (RCTs) for inclusion to minimise risk of bias.

\section{METHODS}

This review is reported in accordance with the Preferred Reporting Items for Systematic Reviews and Meta-Analyses statement 2009. ${ }^{27}$

\section{Literature search and selection of studies}

Any identified study was included if it was a placebo-controlled RCT involving participants with KOA according to the American College of Rheumatology tool and/or a radiographic inspection with the Kellgren/Lawrence (K/L) criteria, in which LLLT was applied to participants' knee(s) and self-reported pain, disability and/or QoL was reported. There were no language restrictions.

We updated a search for eligible articles indexed in PubMed, Embase, Cumulative Index to Nursing and Allied Health Literature, Physiotherapy Evidence Database and Cochrane Central Register of Controlled Trials on 18 February 2019. The database search strings contained synonyms for LLLT and KOA, and keywords were added when optional. The PubMed search string is available in the online supplementary material. The search was continued by reading reference lists of all the eligible trial and relevant review articles, ${ }^{81728}$ citations $^{29-33}$ and a laser book ${ }^{34}$ and involving experts in the field.

Two reviewers (MBS and JMB) each independently selected the trial articles. Both reviewers scrutinised the titles/abstracts of all the publications identified in the search, and any accessible full-text article was retrieved if it was judged potential eligible by at least one reviewer. Both reviewers evaluated the full texts of all potentially eligible retrieved articles and made an independent decision to include or exclude each article, with close attention to the inclusion criteria. When selection disagreements could not be resolved by discussion, a third reviewer (IFN) made the final consensus-based decision. Any retrieved article not fulfilling the inclusion criteria was omitted and listed with reason for exclusion.

\section{Risk-of-bias analysis}

Two reviewers (MBS and JJ) each independently evaluated all included trials for risk of bias at the outcome level, using the Cochrane Collaboration's risk-of-bias tool. ${ }^{35}$ When risk-of-bias disagreements could not be resolved by discussion, a third reviewer (IFN) made the final consensus-based decision. Likelihood of publication bias was assessed with graphical funnel plots. ${ }^{35}$ 


\section{Data extraction and meta-analysis}

Three reviewers (MBS, JMB and KVF) each independently extracted the data for meta-analysis. Two of the reviewers (MBS and KVF) each independently collected the other trial characteristics. The data-extraction forms were subsequently compared, and data disagreements were resolved by consensus-based discussions. Summary data were extracted, unless published individual participant data were available. ${ }^{21}$ The results from the included trials for statistical analysis were selected from outcome scales in adherence to hierarchies published by Juhl et $a .^{36}$

Pain intensity was the primary outcome. As pain reported with continuous, numeric and categorical/ Likert scales highly correlates with pain measured using the VAS, the scores of all pain scales were transformed to 0\%-100\%, corresponding to $0-100 \mathrm{~mm} \mathrm{VAS}^{37}$ The pain results were combined with the mean difference (MD) method, primarily using change scores, that is, when only final scores could be obtained from a trial, change and final scores were mixed in the analysis, since the MD method allows for this without introducing bias. ${ }^{35}$

Self-reported disability results were synthesised with the standardised mean difference (SMD) method using change scores solely. The SMD was adjusted to Hedges' $g$ and interpreted as follows: SMDs of $0.2, \sim 0.5$ and $>0.8$ represent a small, moderate and large effect, respectively. ${ }^{35}$

Lack of QoL data prohibited an analysis of this outcome.

Random effects meta-analyses were conducted, and impact from heterogeneity (inconsistency) on the analyses was examined using $\mathrm{I}^{2}$ statistics. An $\mathrm{I}^{2}$ value of $0 \%$ indicates no inconsistency, and an $\mathrm{I}^{2}$ value of $100 \%$ indicates maximal inconsistency ${ }^{35}$; the values were categorised as low $(25 \%)$, moderate $(50 \%)$ and high $(75 \%){ }^{38}$

SDs for analysis were extracted or estimated from other variance data in a prespecified prioritised order: (1) SD, (2) SE, (3) 95\% CI, (4) p value, (5) IQR, (6) median of correlations, (7) visually from graph or (8) other methods. $^{35}$

The trials were subgrouped by adherence and non-adherence to the WALT recommendations for laser dose per treatment spot, as prespecified. WALT recommends irradiating the knee joint line/synovia with the following doses per treatment spot: $\geq 4 \mathrm{~J}$ using 5-500 mW mean power 780-860 $\mathrm{nm}$ wavelength laser and/or $\geq 1 \mathrm{~J}$ using 5-500 $\mathrm{mW}$ mean power (>1000 $\mathrm{mW}$ peak power) $904 \mathrm{~nm}$ wavelength laser. ${ }^{24} 25$

The main meta-analyses were conducted using two prespecified time points of assessment, that is, immediately after the end of LLLT and last time point of assessment 1-12 weeks after the end of LLLT (follow-up).

MBS performed the meta-analyses, under supervision of JMB, using the software programme Excel 2016 (Microsoft) and Review Manager Version V.5.3 (Copenhagen: The Nordic Cochrane Centre, The Cochrane Collaboration, 2014).
2735 records were initially identified 579 in PubMed 1008 in Embase 221 in CINAHL

98 in PEDro

827 in CENTRAL

2 from experts in the field

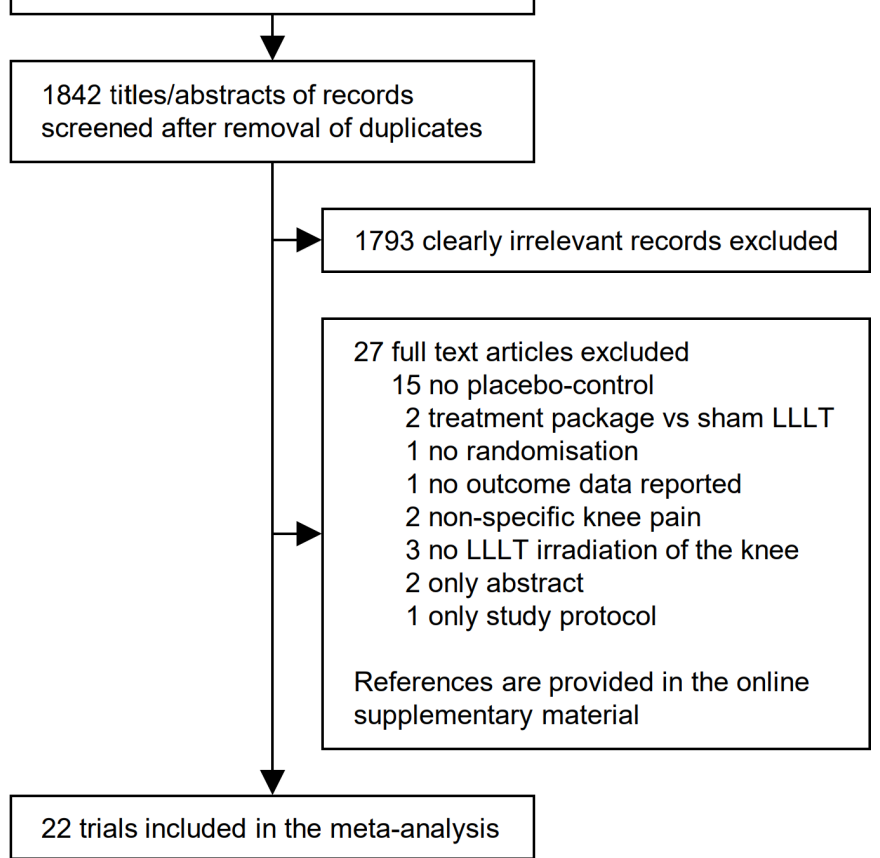

Figure 1 Flow chart illustrating the trial identification process. CENTRAL, Cochrane Central Register of Controlled Trials; CINAHL, Cumulative Index to Nursing and Allied Health Literature; LLLT, low-level laser therapy; PEDro, Physiotherapy Evidence Database.

\section{Patient and public involvement}

Patients or the public were not involved in the conceptualisation or carrying out of this research.

\section{RESULTS}

In total, 2735 records were identified in the search, of which 22 trial articles were judged eligible and included in the review $(n=1089$; figure 1 and tables $1-2)$ with data for meta-analysis $(n=1063)$. Four included trials were not reported in the English language 19212339 and one included trial was unpublished (Gur and Oktayoglu). Excluded articles initially judged potentially eligible were listed with reasons for omission (online supplementary material).

At the group level, the mean age of the participants was 60.25 (50.11-69) years (data from 19 trials), the mean percentage of women was $69.63 \%$ (0-100\%; data from 17 trials), the mean body mass index of the participants was 29.55 (25.8-38; data from 14 trials), the mean of median $\mathrm{K} / \mathrm{L}$ grades was 2.37 (data from 13 trials) and the mean baseline pain was 63.61 mm VAS (35.25-92) (data from 22 trials). LLLT was used as an adjunct to exercise therapy in 11 trials. The mean duration of the treatment periods was 3.53 weeks with the recommended LLLT doses and 3.7 
Table 1 Characteristics of the included trials

\begin{tabular}{|c|c|c|c|c|}
\hline First author & $\begin{array}{l}\text { Intervention group at } \\
\text { baseline }\end{array}$ & $\begin{array}{l}\text { Control group at } \\
\text { baseline }\end{array}$ & $\begin{array}{l}\text { Intervention versus control } \\
\text { programme }\end{array}$ & $\begin{array}{l}\text { Outcome scales, week of } \\
\text { reassessment }\end{array}$ \\
\hline Al Rashoud $2014^{31}$ & $\begin{array}{l}\text { N: } 26 \\
\text { Women: } 62 \% \\
\text { Age: } 52 \text { years } \\
\text { BMI: } 38 \\
\text { VAS pain: } 64 \mathrm{~mm} \\
\text { K/L: - }\end{array}$ & $\begin{array}{l}\text { N: } 23 \\
\text { Women: } 65 \% \\
\text { Age: } 56 \text { years } \\
\text { BMI: } 37.1 \\
\text { VAS pain: } 59 \mathrm{~mm} \\
\text { K/L: - }\end{array}$ & $\begin{array}{l}3 \text { weeks of exercise therapy, advice } \\
\text { and LLLT versus } 3 \text { weeks of exercise } \\
\text { therapy, advice and sham LLLT }\end{array}$ & $\begin{array}{l}\text { Pain: VAS (movement) } \\
\text { Disability: SKFS } \\
\text { QoL: - } \\
\text { Week of assessment: } 2, \mathbf{3 , 9} \text {, } \\
29\end{array}$ \\
\hline Alfredo $2011 / 2018^{29} 52$ & $\begin{array}{l}\text { N: } 24 \\
\text { Women: } 75 \% \\
\text { Age: } 61.15 \text { years } \\
\text { BMI: } 30.16 \\
\text { VAS pain: } 53.2 \mathrm{~mm} \\
\text { K/L: } 3\end{array}$ & $\begin{array}{l}\mathrm{N}: 22 \\
\text { Women: } 80 \% \\
\text { Age: } 62.25 \text { years } \\
\text { BMI: } 29.21 \\
\text { VAS pain: } 35.4 \mathrm{~mm} \\
\text { K/L: } 2\end{array}$ & $\begin{array}{l}3 \text { weeks of LLLT followed by } 8 \text { weeks } \\
\text { of exercise therapy versus } 3 \text { weeks } \\
\text { of sham LLLT followed by } 8 \text { weeks of } \\
\text { exercise therapy }\end{array}$ & $\begin{array}{l}\text { Pain: WOMAC } \\
\text { Disability: WOMAC } \\
\text { QoL: - } \\
\text { Week of assessment: } \mathbf{3}, \mathbf{1 1} \\
24,37\end{array}$ \\
\hline Alghadir $2014^{32}$ & $\begin{array}{l}\mathrm{N}: 20 \\
\text { Women: } 50 \% \\
\text { Age: } 55.2 \text { years } \\
\text { BMI: } 32.34 \\
\text { VAS pain: } 74.5 \mathrm{~mm} \\
\text { K/L: } 2\end{array}$ & $\begin{array}{l}\text { N: } 20 \\
\text { Women: } 40 \% \\
\text { Age: } 57 \text { years } \\
\text { BMI: } 33.09 \\
\text { VAS pain: } 75.5 \mathrm{~mm} \\
\text { K/L: } 2\end{array}$ & $\begin{array}{l}4 \text { weeks of exercise therapy, heat } \\
\text { packs and LLLT versus } 4 \text { weeks of } \\
\text { exercise therapy, heat packs and } \\
\text { sham LLLT }\end{array}$ & $\begin{array}{l}\text { Pain: WOMAC } \\
\text { Disability: WOMAC } \\
\text { QoL: - } \\
\text { Week of assessment: } 4\end{array}$ \\
\hline Bagheri $2011^{23}$ & $\begin{array}{l}\text { N: } 18 \\
\text { Women: } 83.13 \% \\
\text { Age: } 58.32 \text { years } \\
\text { BMI: } 28.87 \\
\text { VAS pain: } 67 \mathrm{~mm} \\
\text { K/L: - }\end{array}$ & $\begin{array}{l}\mathrm{N}: 18 \\
\text { Women: } 83.13 \% \\
\text { Age: } 56.14 \text { years } \\
\text { BMI: } 27.66 \\
\text { VAS pain: } 59 \mathrm{~mm} \\
\text { K/L: - }\end{array}$ & $\begin{array}{l}2 \text { weeks of exercise therapy, } \\
\text { therapeutic ultrasound, TENS and } \\
\text { LLLT versus } 2 \text { weeks of exercise } \\
\text { therapy, therapeutic ultrasound, } \\
\text { TENS and sham LLLT }\end{array}$ & $\begin{array}{l}\text { Pain: WOMAC (VAS) 0-100 } \\
\text { Disability: WOMAC } \\
\text { QoL: - } \\
\text { Week of assessment: } 2\end{array}$ \\
\hline Bülow $1994^{20}$ & $\begin{array}{l}\text { N: } 14 \\
\text { Women: - } \\
\text { Age: - } \\
\text { BMI: - } \\
\text { VAS pain: } 65.08 \mathrm{~mm} \\
\text { K/L: - }\end{array}$ & $\begin{array}{l}\text { N: } 15 \\
\text { Women: - } \\
\text { Age: - } \\
\text { BMl: - } \\
\text { VAS pain: } 56.35 \mathrm{~mm} \\
\text { K/L: - }\end{array}$ & $\begin{array}{l}3 \text { weeks of LLLT versus } 3 \text { weeks of } \\
\text { sham LLLT }\end{array}$ & $\begin{array}{l}\text { Pain: 0-121 Likert scale } \\
\text { (movement/rest) } \\
\text { Disability: - } \\
\text { QoL: - } \\
\text { Week of assessment: } \mathbf{3 , 6}\end{array}$ \\
\hline Delkhosh $2018^{39}$ & $\begin{array}{l}\text { N: } 15 \\
\text { Women: } 100 \% \\
\text { Age: } 55.9 \text { years } \\
\text { BMI: } 26.5 \\
\text { VAS pain: } 57 \mathrm{~mm} \\
\text { K/L: - }\end{array}$ & $\begin{array}{l}\mathrm{N}: 15 \\
\text { Women: } 100 \% \\
\text { Age: } 58.3 \text { years } \\
\text { BMI: } 27.8 \\
\text { VAS pain: } 45 \mathrm{~mm} \\
\text { K/L: - }\end{array}$ & $\begin{array}{l}2 \text { weeks of exercise therapy, } \\
\text { therapeutic ultrasound, TENS and } \\
\text { LLLT versus } 2 \text { weeks of exercise } \\
\text { therapy, therapeutic ultrasound, } \\
\text { TENS and sham LLLT }\end{array}$ & $\begin{array}{l}\text { Pain: VAS } \\
\text { Disability: WOMAC } \\
\text { QoL: - } \\
\text { Week of assessment: } \mathbf{2 , 8}\end{array}$ \\
\hline Fukuda $2011^{30}$ & $\begin{array}{l}\text { N: } 25 \\
\text { Women: } 80 \% \\
\text { Age: } 63 \text { years } \\
\text { BMI: } 30 \\
\text { VAS pain: } 61 \mathrm{~mm} \\
\text { K/L: } 2\end{array}$ & $\begin{array}{l}\text { N: } 22 \\
\text { Women: } 64 \% \\
\text { Age: } 63 \text { years } \\
\text { BMI: } 30 \\
\text { VAS pain: } 62 \mathrm{~mm} \\
\text { K/L: } 2\end{array}$ & $\begin{array}{l}3 \text { weeks of LLLT versus } 3 \text { weeks of } \\
\text { sham LLLT }\end{array}$ & $\begin{array}{l}\text { Pain: VNSP (movement) } \\
\text { Disability: Lequesne } \\
\text { QoL: - } \\
\text { Week of assessment: } 3\end{array}$ \\
\hline Gur $2003^{33}(1.5 \mathrm{~J})$ & $\begin{array}{l}\text { N: } 30 \\
\text { Women: } 83.3 \% \\
\text { Age: } 58.64 \text { years } \\
\text { BMI: } 31.17 \\
\text { VAS pain: } 73.2 \mathrm{~mm} \\
\text { K/L: } 2\end{array}$ & $\begin{array}{l}\text { N: } 30 \\
\text { Women: } 80 \% \\
\text { Age: } 60.52 \text { years } \\
\text { BMI: } 30.27 \\
\text { VAS pain: } 67.4 \mathrm{~mm} \\
\text { K/L: } 2\end{array}$ & $\begin{array}{l}14 \text { weeks of exercise therapy and } \\
2 \text { weeks of LLLT versus } 14 \text { weeks } \\
\text { of exercise therapy and } 2 \text { weeks of } \\
\text { sham LLLT }\end{array}$ & $\begin{array}{l}\text { Pain: VAS (movement) } \\
\text { Disability: - } \\
\text { QoL: - } \\
\text { Week of assessment: } 6,10,14\end{array}$ \\
\hline Gur $2003^{33}(1 \mathrm{~J})$ & $\begin{array}{l}\text { N: } 30 \\
\text { Women: } 76.7 \% \\
\text { Age: } 59.8 \text { years } \\
\text { BMI: } 28.49 \\
\text { VAS pain: } 74.4 \mathrm{~mm} \\
\text { K/L: } 2\end{array}$ & $\begin{array}{l}\text { N: } 30 \\
\text { Women: } 80 \% \\
\text { Age: } 60.52 \text { years } \\
\text { BMI: } 30.27 \\
\text { VAS pain: } 67.4 \mathrm{~mm} \\
\text { K/L: } 2\end{array}$ & $\begin{array}{l}14 \text { weeks of exercise therapy and } \\
2 \text { weeks of LLLT versus } 14 \text { weeks } \\
\text { of exercise therapy and } 2 \text { weeks of } \\
\text { sham LLLT }\end{array}$ & $\begin{array}{l}\text { Pain: VAS (movement) } \\
\text { Disability: - } \\
\text { QoL: - } \\
\text { Week of assessment: } 6,10,14\end{array}$ \\
\hline Gur and Oktayoglu & $\begin{array}{l}\text { N: } 40 \\
\text { Women: } 75 \% \\
\text { Age: } 58.2 \text { years } \\
\text { BMI: } 29.11 \\
\text { VAS pain: } 88 \mathrm{~mm} \\
\text { K/L: } 3\end{array}$ & $\begin{array}{l}\text { N: } 40 \\
\text { Women: } 72.5 \% \\
\text { Age: } 58.26 \text { years } \\
\text { BMI: } 30.11 \\
\text { VAS pain: } 92 \mathrm{~mm} \\
\text { K/L: } 3\end{array}$ & $\begin{array}{l}14 \text { weeks of exercise therapy and } \\
2 \text { weeks of LLLT versus } 14 \text { weeks } \\
\text { of exercise therapy and } 2 \text { weeks of } \\
\text { sham LLLT }\end{array}$ & $\begin{array}{l}\text { Pain: VAS (movement) } \\
\text { Disability: - } \\
\text { QoL: - } \\
\text { Week of assessment: 6, 10, } 14\end{array}$ \\
\hline
\end{tabular}


Table 1 Continued

\begin{tabular}{|c|c|c|c|c|}
\hline First author & $\begin{array}{l}\text { Intervention group at } \\
\text { baseline }\end{array}$ & $\begin{array}{l}\text { Control group at } \\
\text { baseline }\end{array}$ & $\begin{array}{l}\text { Intervention versus control } \\
\text { programme }\end{array}$ & $\begin{array}{l}\text { Outcome scales, week of } \\
\text { reassessment }\end{array}$ \\
\hline Gworys $2012^{18}$ & $\begin{array}{l}\text { N: } 34 \\
\text { Women: - } \\
\text { Age: } 57.6 \\
\text { BMl: - } \\
\text { VAS pain: } 54 \text { mm } \\
\text { K/L: - }\end{array}$ & $\begin{array}{l}\text { N: } 31 \\
\text { Women: - } \\
\text { Age: } 67.7 \\
\text { BMI: - } \\
\text { VAS pain: - } \\
\text { K/L: - }\end{array}$ & $\begin{array}{l}2 \text { weeks of LLLT versus } 2 \text { weeks of } \\
\text { sham LLLT }\end{array}$ & $\begin{array}{l}\text { Pain: VAS } \\
\text { Disability: Lequesne } \\
\text { QoL: - } \\
\text { Week of assessment: } 2\end{array}$ \\
\hline Helianthi $2016^{54}$ & $\begin{array}{l}\text { N: } 30 \\
\text { Women: } 60 \% \\
\text { Age: } 69 \text { years } \\
\text { BMI: } 25.8 \\
\text { VAS pain: } 60.2 \mathrm{~mm} \\
\text { K/L: } 3\end{array}$ & $\begin{array}{l}\text { N: } 29 \\
\text { Women: } 82.8 \% \\
\text { Age: } 68 \text { years } \\
\text { BMI: } 26.3 \\
\text { VAS pain: } 54.1 \mathrm{~mm} \\
\text { K/L: } 3\end{array}$ & $\begin{array}{l}5 \text { weeks of LLLT versus } 5 \text { weeks of } \\
\text { sham LLLT }\end{array}$ & $\begin{array}{l}\text { Pain: VAS (movement) } \\
\text { Disability: Lequesne } \\
\text { QoL: - } \\
\text { Week of assessment: } 2, \mathbf{5}, \mathbf{7}\end{array}$ \\
\hline Hinman $2014^{41}$ & $\begin{array}{l}\mathrm{N}: 71 \\
\text { Women: } 39 \% \\
\text { Age: } 63.4 \text { years } \\
\text { BMI: } 30.7 \\
\text { VAS pain: } 41.5 \mathrm{~mm} \\
\text { K/L: - }\end{array}$ & $\begin{array}{l}\text { N: } 70 \\
\text { Women: } 56 \% \\
\text { Age: } 63.8 \text { years } \\
\text { BMl: } 28.8 \\
\text { VAS pain: } 43 \mathrm{~mm} \\
\text { K/L: - }\end{array}$ & $\begin{array}{l}12 \text { weeks of LLLT versus } 12 \text { weeks of } \\
\text { sham LLLT }\end{array}$ & $\begin{array}{l}\text { Pain: WOMAC } \\
\text { Disability: WOMAC } \\
\text { QoL: AQoL-6D } \\
\text { Week of assessment: 12, } 52\end{array}$ \\
\hline Jensen $1987^{21}$ & $\begin{array}{l}\text { N: } 13 \\
\text { Women: - } \\
\text { Age: - } \\
\text { BMl: - } \\
\text { VAS pain: } 67 \text { mm } \\
\text { K/L: - }\end{array}$ & $\begin{array}{l}\text { N: } 16 \\
\text { Women: - } \\
\text { Age: - } \\
\text { BMI: - } \\
\text { VAS pain: } 72.6 \text { mm } \\
\text { K/L: - }\end{array}$ & $\begin{array}{l}1 \text { week of LLLT versus } 1 \text { week of } \\
\text { sham LLLT }\end{array}$ & $\begin{array}{l}\text { Pain: 0-21 (movement) } \\
\text { Disability: - } \\
\text { QoL: - } \\
\text { Week of assessment: } 1\end{array}$ \\
\hline Mohammed $2018^{56}$ & $\begin{array}{l}\text { N: } 20 \\
\text { Women: } 85 \% \\
\text { Age: } 55.25 \text { years } \\
\text { BMl: } \geq 25 \\
\text { VAS pain: } 70 \mathrm{~mm} \\
\text { K/L: } 2\end{array}$ & $\begin{array}{l}\text { N: } 20 \\
\text { Women: } 85 \% \\
\text { Age: } 50.11 \text { years } \\
\text { BMl: } \geq 25 \\
\text { VAS pain: } 80 \mathrm{~mm} \\
\text { K/L: } 2\end{array}$ & $\begin{array}{l}4 \text { weeks of LLLT versus } 4 \text { weeks of } \\
\text { sham LLLT }\end{array}$ & $\begin{array}{l}\text { Pain: VAS } \\
\text { Disability: - } \\
\text { QoL: - } \\
\text { Week of assessment: } 4\end{array}$ \\
\hline Nambi $2016^{48}$ & $\begin{array}{l}\text { N: } 17 \\
\text { Women: - } \\
\text { Age: } 58 \\
\text { BMl: } 26.9 \\
\text { VAS pain: } 78 \text { mm } \\
\text { K/L: } 3.1\end{array}$ & $\begin{array}{l}\text { N: } 17 \\
\text { Women: - } \\
\text { Age: } 60 \\
\text { BMl: } 28.3 \\
\text { VAS pain: } 76 \text { mm } \\
\text { K/L: } 3.2\end{array}$ & $\begin{array}{l}4 \text { weeks of exercise therapy, kinesio } \\
\text { tape and LLLT versus } 4 \text { weeks of } \\
\text { exercise therapy, kinesio tape and } \\
\text { sham LLLT }\end{array}$ & $\begin{array}{l}\text { Pain: VAS } \\
\text { Disability: - } \\
\text { QoL: - } \\
\text { Week of assessment: } \mathbf{4 , 8}\end{array}$ \\
\hline Nivbrant $1992^{19}$ & $\begin{array}{l}\text { N: } 15 \\
\text { Women: } 69.2 \% \\
\text { Age: } 69 \text { years } \\
\text { BMl: - } \\
\text { VAS pain: } 67 \mathrm{~mm} \\
\text { K/L: - }\end{array}$ & $\begin{array}{l}\text { N: } 15 \\
\text { Women: } 84.6 \% \\
\text { Age: } 66 \text { years } \\
\text { BMl: - } \\
\text { VAS pain: } 58 \mathrm{~mm} \\
\text { K/L: - }\end{array}$ & $\begin{array}{l}2 \text { weeks of LLLT versus } 2 \text { weeks of } \\
\text { sham LLLT }\end{array}$ & $\begin{array}{l}\text { Pain: VAS (movement) } \\
\text { Disability: Walking disability } \\
\text { QoL: - } \\
\text { Week of assessment: 2, 3, } 6\end{array}$ \\
\hline
\end{tabular}

Continued 


\begin{tabular}{|c|c|c|c|c|}
\hline First author & $\begin{array}{l}\text { Intervention group at } \\
\text { baseline }\end{array}$ & $\begin{array}{l}\text { Control group at } \\
\text { baseline }\end{array}$ & $\begin{array}{l}\text { Intervention versus control } \\
\text { programme }\end{array}$ & $\begin{array}{l}\text { Outcome scales, week of } \\
\text { reassessment }\end{array}$ \\
\hline Tascioglu $2004^{40}(3 \mathrm{~J})$ & $\begin{array}{l}\text { N: } 20 \\
\text { Women: } 70 \% \\
\text { Age: } 62.86 \text { years } \\
\text { BMI: } 27.56 \\
\text { VAS pain: } 68 \mathrm{~mm} \\
\text { K/L: } 2\end{array}$ & $\begin{array}{l}\mathrm{N}: 20 \\
\text { Women: } 65 \% \\
\text { Age: } 64.27 \text { years } \\
\text { BMl: } 29.56 \\
\text { VAS pain: } 63.88 \mathrm{~mm} \\
\text { K/L: } 2\end{array}$ & $\begin{array}{l}2 \text { weeks of LLLT versus } 2 \text { weeks of } \\
\text { sham LLLT }\end{array}$ & $\begin{array}{l}\text { Pain: WOMAC } \\
\text { Disability: WOMAC } \\
\text { QoL: - } \\
\text { Week of assessment: } \mathbf{3 , 2 6}\end{array}$ \\
\hline $\begin{array}{l}\text { Youssef } 2016^{42} \text { (904 } \\
n m)\end{array}$ & $\begin{array}{l}\text { N: } 18 \\
\text { Women: } 66.7 \% \\
\text { Age: } 67.5 \\
\text { BMI:<40 } \\
\text { VAS pain: } 51.67 \mathrm{~mm} \\
\text { K/L: } 2\end{array}$ & $\begin{array}{l}\mathrm{N}: 15 \\
\text { Women: } 66.7 \% \\
\text { Age: } 66.3 \text { years } \\
\text { BMI: }<40 \\
\text { VAS pain: } 50 \mathrm{~mm} \\
\text { K/L: } 2\end{array}$ & $\begin{array}{l}8 \text { weeks of exercise therapy and LLLT } \\
\text { versus } 8 \text { weeks of exercise therapy } \\
\text { and sham LLLT }\end{array}$ & $\begin{array}{l}\text { Pain: WOMAC } \\
\text { Disability: WOMAC } \\
\text { QoL: - } \\
\text { Week of assessment: } 8\end{array}$ \\
\hline $\begin{array}{l}\text { Youssef } 2016^{42}(880 \\
n m)\end{array}$ & $\begin{array}{l}\text { N: } 18 \\
\text { Women: } 61.1 \% \\
\text { Age: } 67.3 \\
\text { BMI: <40 } \\
\text { VAS pain: } 52.50 \mathrm{~mm} \\
\text { K/L: } 2\end{array}$ & $\begin{array}{l}\text { N: } 15 \\
\text { Women: } 66.7 \% \\
\text { Age: } 66.3 \text { years } \\
\text { BMI: }<40 \\
\text { VAS pain: } 50 \mathrm{~mm} \\
\text { K/L: } 2\end{array}$ & $\begin{array}{l}8 \text { weeks of exercise therapy and LLLT } \\
\text { versus } 8 \text { weeks of exercise therapy } \\
\text { and sham LLLT }\end{array}$ & $\begin{array}{l}\text { Pain: WOMAC } \\
\text { Disability: WOMAC } \\
\text { QoL: - } \\
\text { Week of assessment: } 8\end{array}$ \\
\hline
\end{tabular}

The values for age and body mass index (BMI) are means and the values for K/L grade are medians. Baseline Visual Analogue Scale (VAS) scores have been extracted or estimated as described in the Method section. Week of assessment in bold denotes time point used for the main metaanalyses.

AQoL-6D, Assessment of Quality of Life 6 Dimensions; DIQ, Disability Index Questionnaire; K/L, Kellgren/Lawrence; LLLT, low-level laser therapy; NRS, Numeric Rating Scale; QoL, quality of life; SKFS, Saudi Knee Function Scale; TENS, Transcutaneous Electrical Nerve Stimulation; VNPS, Visual Numerical Pain Scale; WOMAC, Western Ontario and McMaster Universities Osteoarthritis Index.

weeks with the non-recommended LLLT doses (tables 1 and 2). Non-recommended LLLT doses were applied in nine of the trials. That is, $\mathrm{Al}$ Rashoud et al, ${ }^{31}$ Bülow et $a l,{ }^{20}$ Tascioglu $e t a l^{40}$ and Bagheri $e t a l^{23}$ applied too few $(<4)$ Joules per treatment spot with $830 \mathrm{~nm}$ wavelength, Jensen et $a l^{21}$ Nivbrant $e t a l^{19}$ and Hinman et $a l^{41}$ applied too few $(<1)$ Joules per treatment spot with $904 \mathrm{~nm}$ wavelength and Youssef $e t a t^{2}$ (one group) and Rayegani $e t a t^{43}$ used continuous laser with too long of a wavelength $(880 \mathrm{~nm}$; table 2). No adverse event was reported by any of the trial authors. None of the trial authors stated receiving funding from the laser industry (online supplementary material).

Overall, pain was significantly reduced by LLLT compared with the placebo control at the end of therapy (14.23 mm VAS (95\% CI 7.31 to 21.14 ); $\mathrm{I}^{2}=93 \%$; $\mathrm{n}=816$; figure 2) and during follow-ups 1-12 weeks later (15.92 mm VAS (95\% CI 6.47 to 25.37); $\mathrm{I}^{2}=93 \%$; $\mathrm{n}=581$; figure 3 ). The dose subgroup analyses demonstrated that pain was significantly reduced by the recommended LLLT doses compared with placebo at the end of therapy $(18.71 \mathrm{~mm}$
(95\% CI 9.42 to 27.99 ); $\mathrm{I}^{2}=95 \%$; $\mathrm{n}=480$; figure 2 ) and during follow-ups 2-12 weeks later (23.23 mm VAS $(95 \%$ CI 10.60 to 35.86 ); $\mathrm{I}^{2}=95 \%$; $\mathrm{n}=392$; figure 3 ). The dose subgroup analyses demonstrated that pain was significantly reduced by the non-recommended LLLT doses compared with placebo at the end of therapy $(6.34 \mathrm{~mm}$ $\operatorname{VAS}\left(95 \%\right.$ CI 1.26 to 11.41 ); $\mathrm{I}^{2}=44 \%$; $\mathrm{n}=336$; figure 2 ), but the difference during follow-ups 1-12 weeks later was not significant ( $6.20 \mathrm{~mm}$ VAS (95\% CI -0.65 to 13.05 ); $\mathrm{I}^{2}=38 \%$; $\mathrm{n}=189$; figure 3 ). The between-subgroup differences (recommended versus non-recommended doses) in pain results were significantly in favour of the recommended LLLT doses regarding both time points $(\mathrm{p}=0.02$ and 0.02 ; figures 2 and 3 ).

Overall, disability was significantly reduced by LLLT compared with placebo at the end of therapy $(\mathrm{SMD}=0.59$ ( $95 \%$ CI 0.33 to 0.86 ); $\mathrm{I}^{2}=57 \%$; $=617$; figure 4 ) and during follow-ups $1-12$ weeks later $(\mathrm{SMD}=0.66(95 \%$ CI 0.23 to $1.09) ; I^{2}=67 \%$; $n=289$; figure 5 ). The dose subgroup analyses demonstrated that disability was significantly reduced by the recommended LLLT doses compared with placebo 
Table 2 Laser therapy characteristics of the included trials

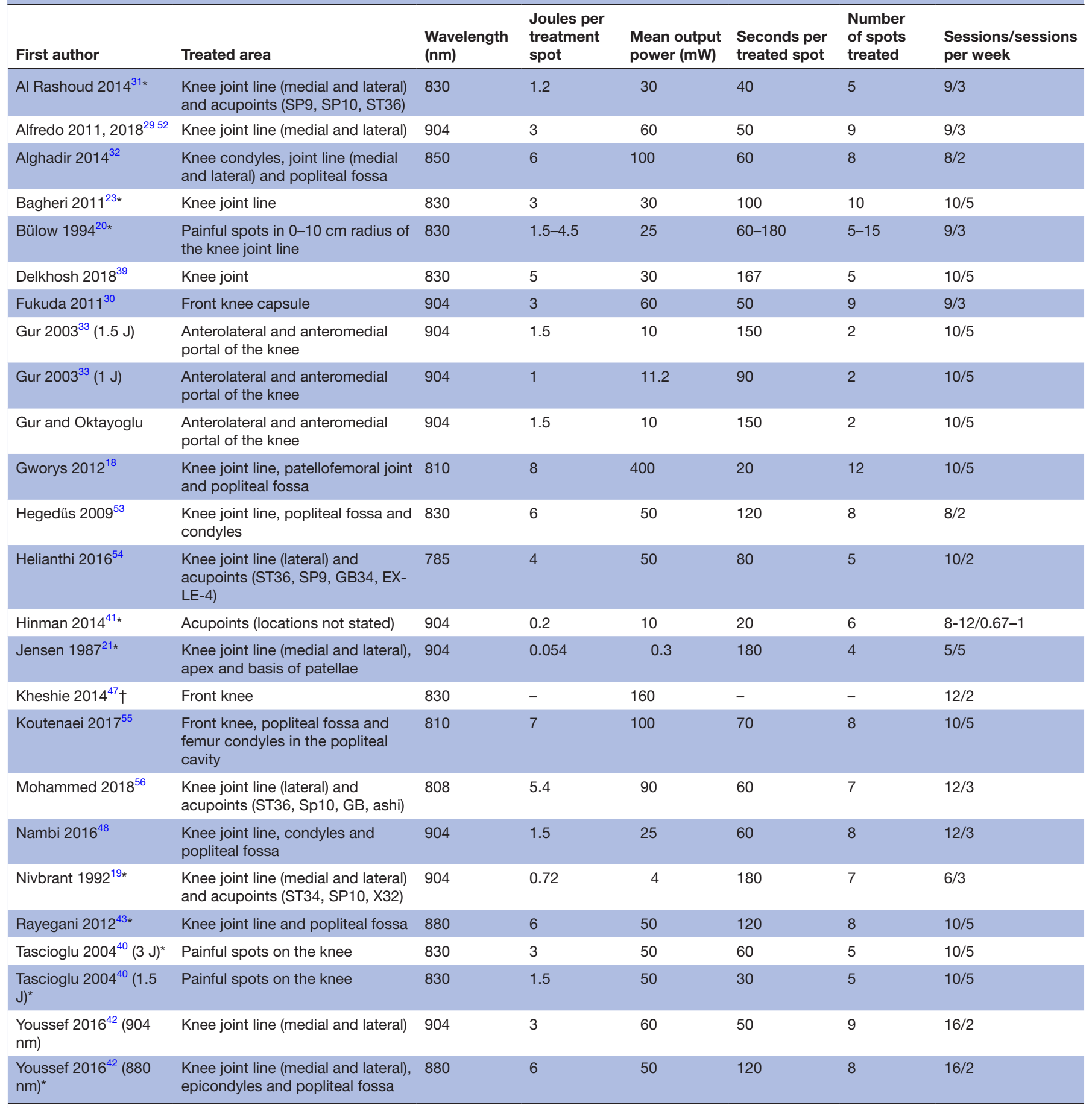

${ }^{*}$ Non-recommended low-level laser therapy dose.

†1250 Joules per session.

at the end of therapy (SMD $=0.75$ (95\% CI 0.46 to 1.03 ); $\mathrm{I}^{2}=34 \% ; n=339$; figure 4 ) and during follow-ups 2-8 weeks later (SMD=1.31 (95\% CI 0.92 to 1.69$) ; \mathrm{I}^{2}=0 \% ; \mathrm{n}=129$; figure 5). The dose subgroup analyses demonstrated that disability was neither significantly reduced by the non-recommended LLLT doses compared with placebo at the end of therapy (SMD $=0.36$ (95\% CI -0.02 to 0.73$)$; $\mathrm{I}^{2}=49 \% ; \mathrm{n}=278$; figure 4) nor during follow-ups $1-12$ weeks later (SMD $=0.26$ (95\% CI -0.06 to 0.58$) ; \mathrm{I}^{2}=0 \%$; $\mathrm{n}=160$; figure 5). The between-subgroup differences in disability results were in favour of the recommended LLLT doses over the non-recommended LLLT doses but only significantly regarding one of two time points ( $\mathrm{p}=0.11$ and $<0.0001$; figures $4-5)$.

No QoL meta-analysis was performed because this outcome was only assessed in a single trial, that is, by Hinman et al who applied a non-recommended LLLT dose and reported insignificant results. ${ }^{41}$ 


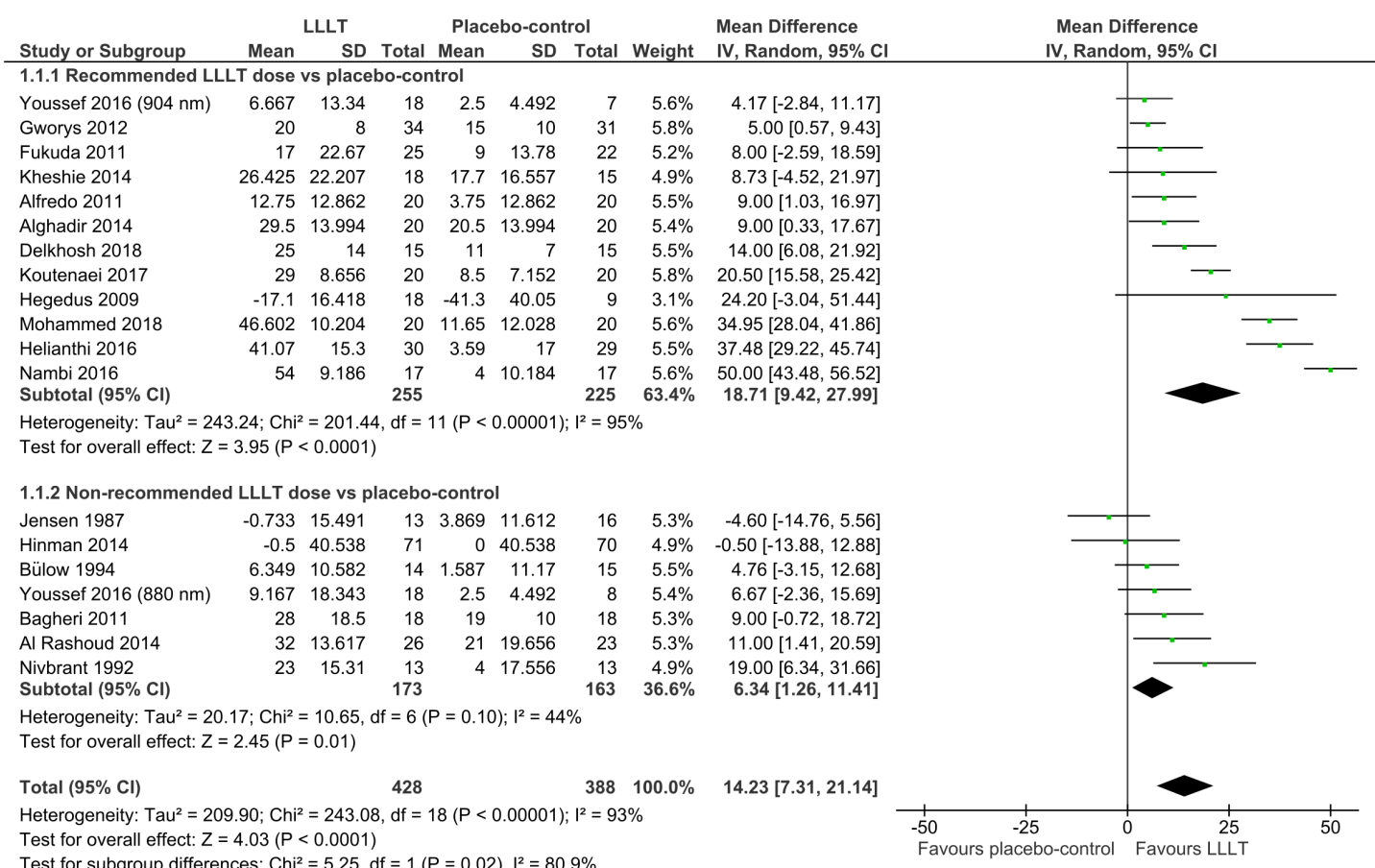

Figure 2 Pain results from immediately after the end of therapy. LLLT, low-level laser therapy.

The funnel plots indicated that there was no publication bias (online supplementary material). We additionally checked for small study bias by reducing the statistical weight of the smallest studies through a change from random to fixed effects models and this led to similar mean effect estimates, indicating that there was no small study bias (online supplementary material)..$^{35}$

Methodological quality of the included trials was judged adequate (low risk of bias), unclear (unclear risk of bias) and inadequate (high risk of bias) in $75 \%, 19 \%$ and $6 \%$ instances, respectively. Risk of detection bias and reporting bias appeared low in all the trials. There was a lack of information regarding random sequence generation in five trials, allocation concealment in 12 trials, blinding of therapist in four trials and incomplete outcome data in four trials. Therapist blinding was inadequate in seven trials and there was an inadequate handling of data in a single trial (figure 6). However, risk-of-bias subgroup analyses conducted post hoc revealed that there was no statistically significant interaction between the effect estimates and risk of bias, and the analyses did not display a drop in statistical heterogeneity (online supplementary material). Support for our risk of bias judgments is available (online supplementary material).

Neither did the levels of statistical heterogeneity change when we switched from the MD to the SMD method post hoc (online supplementary material).

Post hoc analyses demonstrated that LLLT was significantly superior to placebo both with exercise therapy ( $p=0.0009$ for pain and $p<0.0001$ for disability) and without exercise therapy $(p=0.01$ for pain and $p=0.008$

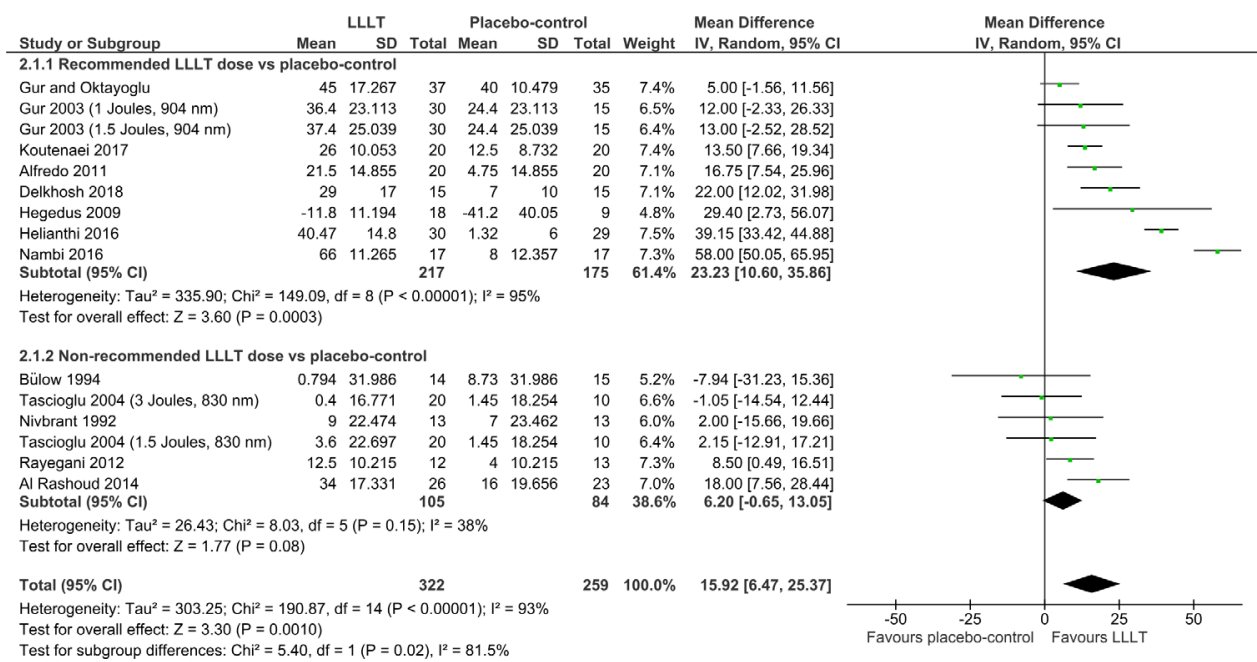

Figure 3 Pain results from follow-ups 1-12 weeks after the end of therapy. LLLT, low-level laser therapy. 


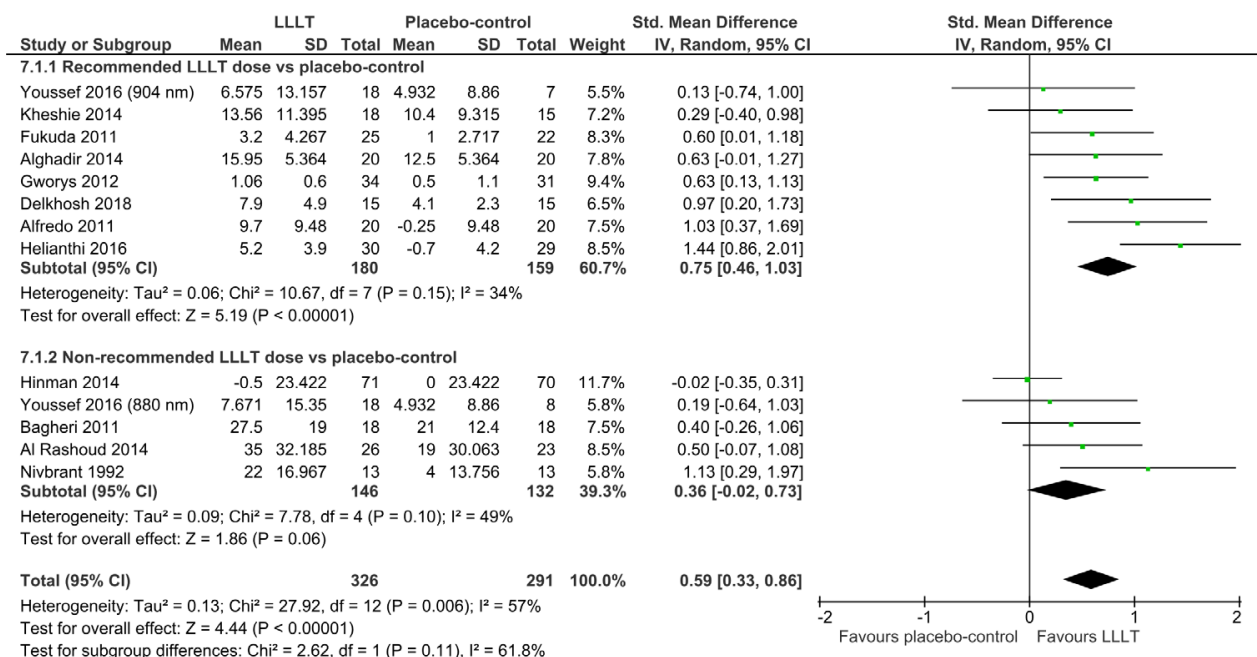

for disability) as cointervention (online supplementary material).

Post hoc analyses were performed to more precisely estimate the pain time-effect profile for the recommended LLLT doses by imputing the results of the trials with these doses in subgroups with narrower time intervals. Pain was significantly reduced by the recommended LLLT doses compared with placebo immediately after therapy weeks 2-3 and 4-8 and at follow-ups 2-4, 6-8 and 12 weeks later; the peak point was 2-4 weeks after the end of therapy (31.87 mm VAS beyond placebo (95\% CI 18.18 to 45.56); $\left.\mathrm{I}^{2}=93 \% ; \mathrm{n}=322\right)$. The 21-week and 34-week follow-up pain results were not statistically significant (figure 7 and online supplementary material). The statistical heterogeneity in the main pain analyses of the recommended LLLT doses was high $\left(I^{2}=95 \%\right.$; figures $\left.2-3\right)$ but the mean statistical heterogeneity of the five subgroups covering the same time period was only moderate $\left(\mathrm{I}^{2}=58 \%\right.$; figure 7 and online supplementary material).

\section{DISCUSSION}

Our meta-analyses showed that pain and disability were significantly reduced by LLLT compared with placebo.
We subgrouped the included trials according to the WALT recommendations (2010) for laser dose per treatment spot, and this revealed a significant dose-response relationship. Our principal finding is that the recommended LLLT doses offer clinically relevant pain relief in KOA. The non-recommended LLLT doses provided no or little positive effect.

The absolute minimally clinically important improvement (MCII) of pain in KOA has been estimated to be 19.9, 17 and 9 units on a $0-100$ scale in 2005, 2012 and 2015 , respectively. ${ }^{44-46}$ It is important to note that the MCII of pain is a within-subject improvement and depends on baseline pain intensity. ${ }^{44-46}$ The pain reduction from the recommended LLLT doses was significantly superior to placebo even at follow-ups 12 weeks after the end of therapy, and the difference was greater than $20 \mathrm{~mm}$ VAS from the final 4-8 weeks of therapy through follow-ups 6-8 weeks after the end of therapy. Interestingly, the pain reduction from the recommended LLLT doses peaked at follow-ups 2-4 weeks after the end of therapy $(31.87 \mathrm{~mm}$ VAS highly significantly beyond placebo).

Disability was also significantly reduced by the recommended LLLT doses compared with placebo, that is, to

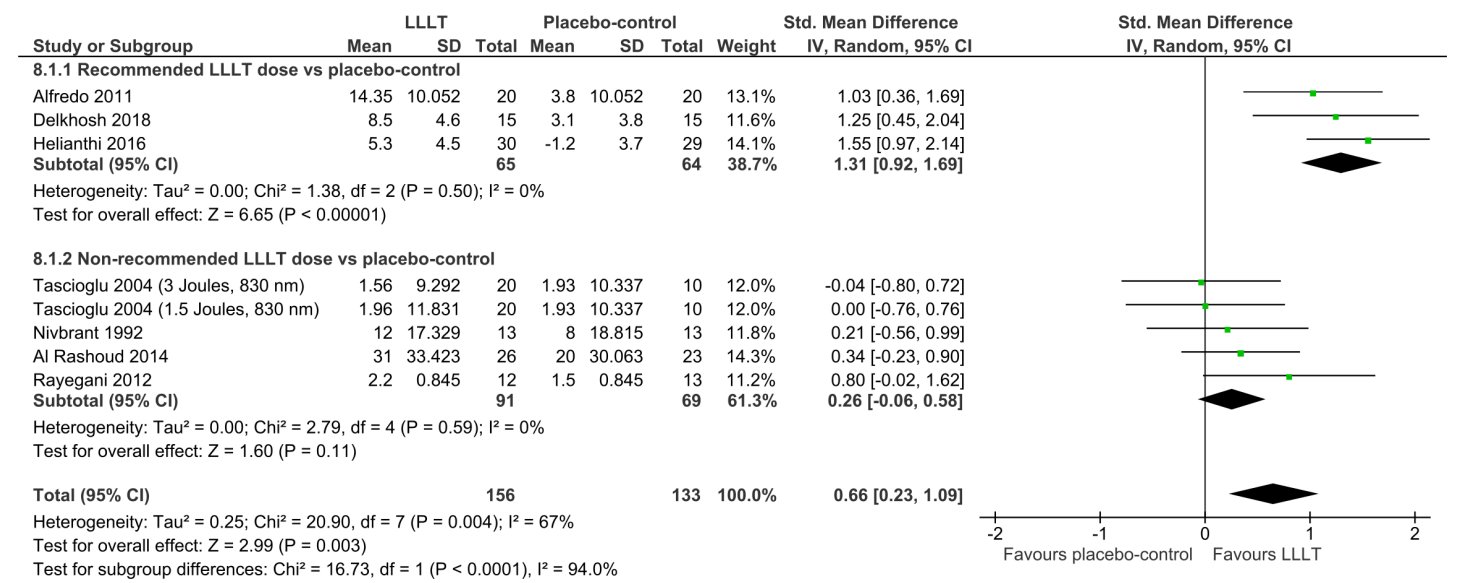

Figure 5 Disability results from follow-ups 1-12 weeks after the end of therapy. LLLT, low-level laser therapy. 


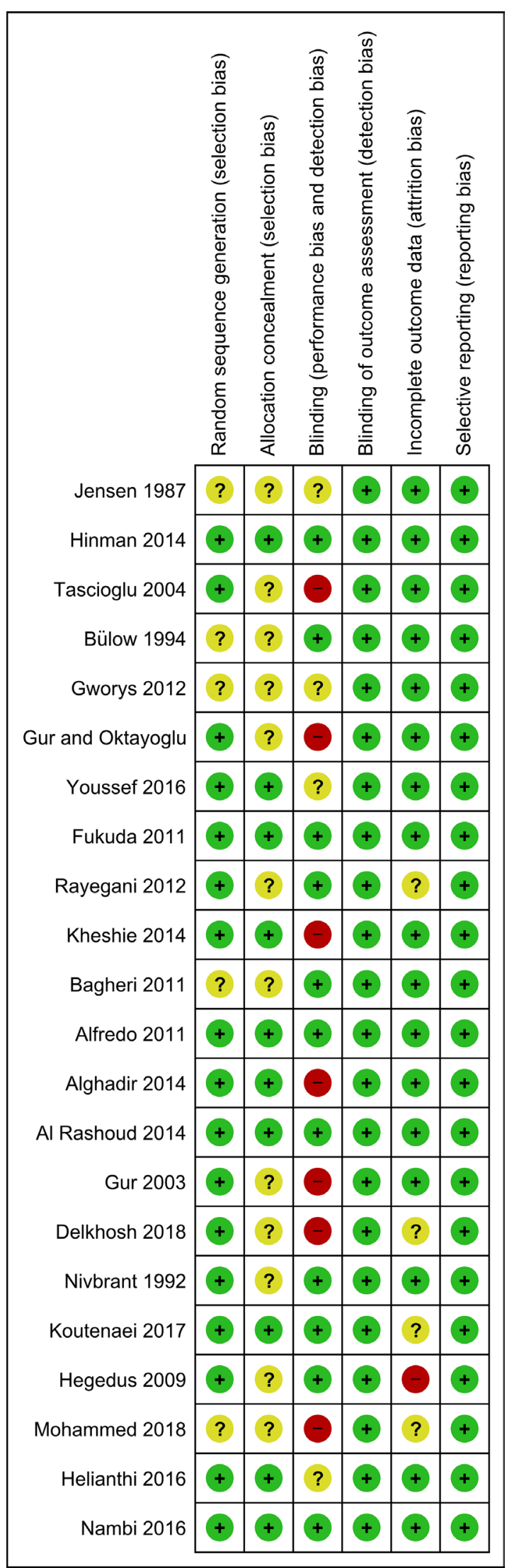

Figure 6 Risk-of-bias plot of the included trials. The trials are ranked by mean pain effect estimates, that is, more laser positive results in the bottom of the figure; the plot is based on the results from the main pain analyses (immediately after the end of therapy, primarily). a moderate extent at the end of therapy $(\mathrm{SMD}=0.75)$ and to a large extent during follow-ups 2-8 weeks later $(\mathrm{SMD}=1.31)$. More trials with disability assessments are needed to precisely estimate the effect of LLLT on this outcome during follow-up.

Furthermore, our analyses demonstrated that LLLT is effective in KOA both with and without exercise therapy as cointervention. Strength training was seemingly only used as an adjunct to LLLT in two of the included trials, ${ }^{478}$ and thus more trials with this combination of treatments are needed.

Risk of bias of the included trials appeared insignificant and could not explain the statistical heterogeneity (online supplementary material). We find it plausible that some of the statistical heterogeneity of the overall analyses is associated with the dose subgroup criteria (wavelength-specific laser doses per treatment spot) since the mean levels of statistical heterogeneity of the subgroup analyses were consistently lower than the overall levels. It is unknown to us whether other differences in the LLLT protocols impacted the results.

The statistical heterogeneity in the main pain analyses of the recommended LLLT doses was high, and some of it can be explained by the pooling of results from various time points of assessment given the pain reduction increased and subsequent decreased with time; the pain reduction time profile showed a drop in statistical heterogeneity to a moderate level.

According to WALT, the osteoarthritic knee should be laser irradiated to reduce inflammation and promote tissue repair. ${ }^{242549}$ One of the discrepancies from our review and previously published reviews of the same topic is that we omitted the RCT by Yurtkuran $e t a l,{ }^{8172850}$ as they solely applied laser to an acupoint located distally from the knee joint (spleen 9).

In line with our findings and the WALT dose recommendations, Joensen $e t a l^{26}$ observed that the percentage of laser penetrating rat skin at 810 and $904 \mathrm{~nm}$ wavelength was $20 \%$ and $38 \%-58 \%$, respectively. That is, to deliver the same dose beneath the skin, 2.4 times the energy on the skin surface is required with an $810 \mathrm{~nm}$ laser compared with a $904 \mathrm{~nm}$ laser device. This may be due to the different wavelengths and/or because 904 $\mathrm{nm}$ laser is superpulsed (pulse peak power $\geq 10000 \mathrm{~mW}$ typically), whereas shorter wavelength laser is delivered continuously or with less intense pulsation. ${ }^{26}$ The estimated median dose applied with the recommended LLLT was 6 and $3 \mathrm{~J}$ per treatment spot with 785-860 and $904 \mathrm{~nm}$ wavelength laser, respectively. Most of the trial authors reported LLLT parameters in detail but did not state whether the laser devices were calibrated. Therefore, in the LLLT trials with non-significant effect estimates, equipment failure cannot be ruled out.

It is important to note that no adverse events were reported by any of the trial authors and the dropout rate was minor, indicating that LLLT is harmless.

Our clinical findings that the effect of LLLT progresses over time is in line with in vivo results of Wang et al. ${ }^{12}$ The 


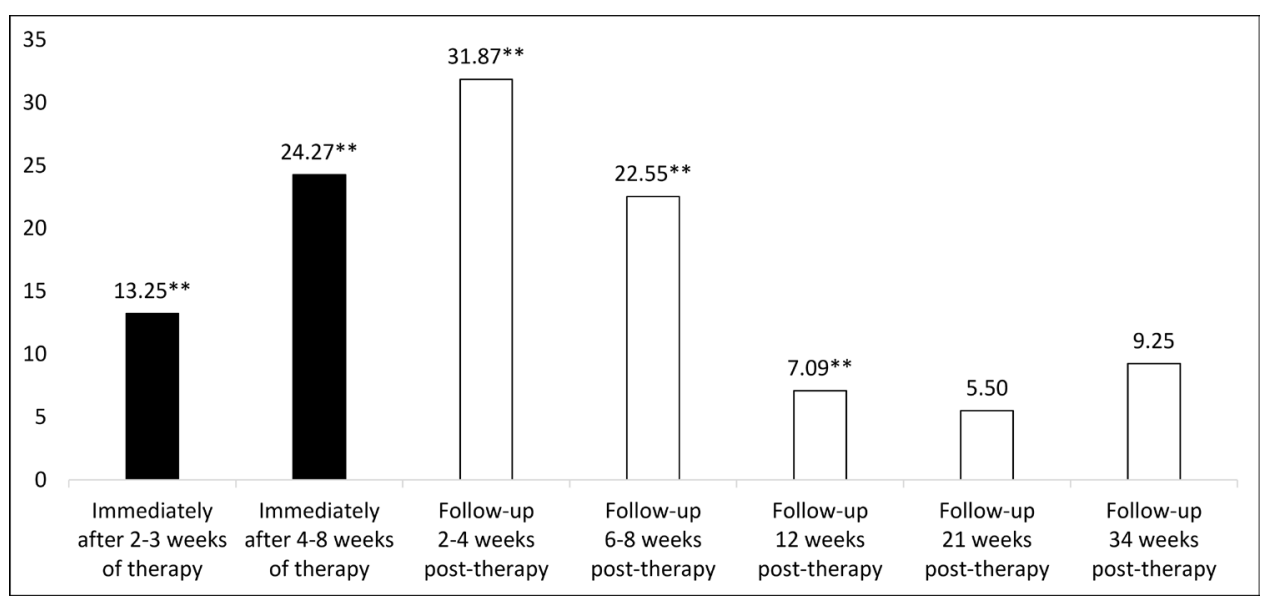

Figure 7 Pain time-effect profile (recommended low-level laser therapy (LLLT) doses versus placebo-control). Values on the $y$-axis are mm Visual Analogue Scale (VAS) pain results. Positive VAS score indicates that the recommended LLLT doses are superior to placebo. The related forest plot is available (online supplementary material). ${ }^{* *}$ The recommended LLLT doses are highly statistically significantly superior to placebo $(p \leq 0.01)$.

positive effect from LLLT seems to last longer than those of widely recommended painkiller drugs. ${ }^{51}$ The effect of using the NSAID tiaprofenic acid, for example, is probably gone within a week, unless the treatment is continued. ${ }^{51}$ Future trials should investigate whether booster sessions of LLLT can prolong the positive effect. Comparative cost-effectiveness analyses of LLLT and NSAIDs would also be of great interest.

\section{Strengths and limitations of this study}

In contrast to previous reviews on the current topic, our review was conducted in conformance with an a priori published protocol, ${ }^{8} 1728$ which included a detailed plan for statistical analysis (eg, laser dose subgroup criteria). Furthermore, this is the first review on this topic without language restrictions, ${ }^{81728}$ and this expansion proved important since four $(18 \%)$ of the included trials were reported in non-English language. ${ }^{19} 212339$

We conducted a series of meta-analyses illustrating the effect of LLLT on pain over time. To ensure high reproducibility of the meta-analyses, three persons each independently extracted the outcome data from the included trial articles.

This review is not without limitations. It lacks QoL analyses, a detailed disability time-effect analysis and direct comparisons between LLLT and other interventions.

\section{CONCLUSIONS}

LLLT reduces pain and disability in KOA at 4-8 J with $785-860 \mathrm{~nm}$ wavelength and at $1-3 \mathrm{~J}$ with $904 \mathrm{~nm}$ wavelength per treatment spot.

Contributors MBS, JMB and HL wrote the PROSPERO protocol. MBS and JMB selected the trials, with the involvement of IFN when necessary. MBS and JJ judged the risk of bias, with the involvement of IFN when necessary. MBS and IFN did the translations. MBS, JMB and KVF extracted the data. MBS performed the analyses, under supervision of JMB. All the authors participated in interpreting of the results. MBS drafted the first version of the manuscript, and subsequently revised it, based on comments by RÁBL-M, HS and all the other authors. All the authors read and accepted the final version of the manuscript.

Funding The University of Bergen funded this research.

Competing interests JMB and RÁBL-M are post-presidents and former board members of World Association for Laser Therapy, a non-for-profit research organization from which they have never received funding, grants or fees. The other authors declared that they had no conflict of interests related to this work.

Patient consent for publication Not required.

Provenance and peer review Not commissioned; externally peer reviewed.

Data availability statement The dataset for meta-analysis is available from the corresponding author upon reasonable request. The corresponding author affirms that the manuscript is an honest, accurate and transparent account of the study being reported; that no important aspects of the study have been omitted; and that any discrepancies from the study as planned (and, if relevant, registered) have been explained.

Open access This is an open access article distributed in accordance with the Creative Commons Attribution Non Commercial (CC BY-NC 4.0) license, which permits others to distribute, remix, adapt, build upon this work non-commercially, and license their derivative works on different terms, provided the original work is properly cited, appropriate credit is given, any changes made indicated, and the use is non-commercial. See: http://creativecommons.org/licenses/by-nc/4.0/.

\section{ORCID iD}

Martin Bjørn Stausholm http://orcid.org/0000-0001-9869-0705

\section{REFERENCES}

1 Heidari B. Knee osteoarthritis prevalence, risk factors, pathogenesis and features: Part I. Caspian J Intern Med 2011;2:205-12.

2 Berenbaum F. Osteoarthritis as an inflammatory disease (osteoarthritis is not osteoarthrosis!). Osteoarthritis Cartilage 2013;21:16-21.

3 Bartels EM, Juhl CB, Christensen R, et al. Aquatic exercise for the treatment of knee and hip osteoarthritis. Cochrane Database Syst Rev 2016;3.

4 Juhl C, Christensen R, Roos EM, et al. Impact of exercise type and dose on pain and disability in knee osteoarthritis: a systematic review and meta-regression analysis of randomized controlled trials. Arthritis Rheumatol 2014;66:622-36.

5 Rannou F, Pelletier J-P, Martel-Pelletier J. Efficacy and safety of topical NSAIDs in the management of osteoarthritis: evidence from real-life setting trials and surveys. Semin Arthritis Rheum 2016;45:S18-21.

6 Bannuru RR, Schmid CH, Kent DM, et al. Comparative effectiveness of pharmacologic interventions for knee osteoarthritis: a systematic review and network meta-analysis. Ann Intern Med 2015;162:46-54. 
7 Bjordal JM, Ljunggren AE, Klovning A, et al. Non-Steroidal antiinflammatory drugs, including cyclo-oxygenase-2 inhibitors, in osteoarthritic knee pain: meta-analysis of randomised placebo controlled trials. BMJ 2004:329.

8 Rayegani SM, Raeissadat SA, Heidari S, et al. Safety and effectiveness of low-level laser therapy in patients with knee osteoarthritis: a systematic review and meta-analysis. J Lasers Med Sci 2017;8:S12-19.

9 Hamblin MR. Can osteoarthritis be treated with light? Arthritis Res Ther 2013;15.

10 Tomazoni SS, Leal-Junior ECP, Pallotta RC, et al. Effects of photobiomodulation therapy, pharmacological therapy, and physical exercise as single and/or combined treatment on the inflammatory response induced by experimental osteoarthritis. Lasers Med Sci 2017;32:101-8

11 Tomazoni SS, Leal-Junior ECP, Frigo L, et al. Isolated and combined effects of photobiomodulation therapy, topical nonsteroidal anti-inflammatory drugs, and physical activity in the treatment of osteoarthritis induced by papain. J Biomed Opt 2016;21:108001.

12 Wang P, Liu C, Yang X, et al. Effects of low-level laser therapy on joint pain, synovitis, anabolic, and catabolic factors in a progressive osteoarthritis rabbit model. Lasers Med Sci 2014;29:1875-85.

13 Assis L, Almeida T, Milares LP, et al. Musculoskeletal atrophy in an experimental model of knee osteoarthritis: the effects of exercise training and low-level laser therapy. Am J Phys Med Rehabil 2015;94:609-16.

14 Pallotta RC, Bjordal JM, Frigo L, et al. Infrared (810-nm) low-level laser therapy on rat experimental knee inflammation. Lasers Med Sci 2012;27:71-8.

15 Geenen R, Overman CL, Christensen R, et al. EULAR recommendations for the health professional's approach to pain management in inflammatory arthritis and osteoarthritis. Ann Rheum Dis 2018;77:797-807.

16 Collins NJ, Hart HF, Mills KAG. Osteoarthritis year in review 2018: rehabilitation and outcomes. Osteoarthritis Cartilage 2019;27:378-91.

17 Huang Z, Chen J, Ma J, et al. Effectiveness of low-level laser therapy in patients with knee osteoarthritis: a systematic review and metaanalysis. Osteoarthritis Cartilage 2015;23:1437-44.

18 Gworys K, Gasztych J, Puzder A, et al. Influence of various laser therapy methods on knee joint pain and function in patients with knee osteoarthritis. Ortop Traumatol Rehabil 2012;14:269-77.

19 Nivbrant B, Friberg S. Laser tycks ha effekt pa knaledsartros men vetenskapligt bevis saknas [Swedish]. Lakartidningen [Journal of the Swedish Medical Association] 1992;89:859-61.

20 Bülow PM, Jensen H, Danneskiold-Samsøe B. Low power Ga-Al-As laser treatment of painful osteoarthritis of the knee. A double-blind placebo-controlled study. Scand J Rehabil Med 1994;26:155-9.

21 Jensen H, Harreby M, Kjer J. Infrarød laser - effekt ved smertende knæartrose? [Danish]. Ugeskr Laeger 1987;149:3104-6.

22 Stausholm MB, Bjordal JM, Lopes-Martins RAB, et al. Methodological flaws in meta-analysis of low-level laser therapy in knee osteoarthritis: a letter to the editor. Osteoarthritis Cartilage 2017;25:e9-10.

23 Bagheri SR, Fatemi E, Fazeli SH, et al. Efficacy of low level laser on knee osteoarthritis treatment [Persian]. Koomesh 2011;12:285-92.

24 WALT. Recommended treatment doses for low level laser therapy 780-860 nm wavelength: world association for laser therapy, 2010 Available: http://waltza.co.za/wp-content/uploads/2012/08/Dose table 780-860nm for Low Level Laser Therapy WALT-2010.pdf

25 WALT. Recommended treatment doses for low level laser therapy $904 \mathrm{~nm}$ wavelength: world association for laser therapy, 2010. Available: http://waltza.co.za/wp-content/uploads/2012/08/Dose_ table_904nm_for_Low_Level_Laser_Therapy_WALT-2010.pdf

26 Joensen J, Øvsthus K, Reed RK, et al. Skin penetration time-profiles for continuous $810 \mathrm{~nm}$ and Superpulsed $904 \mathrm{~nm}$ lasers in a rat model. Photomed Laser Surg 2012;30:688-94.

27 Moher D, Liberati A, Tetzlaff J, et al. Preferred reporting items for systematic reviews and meta-analyses: the PRISMA statement. PLOS Med 2009;6:e1000097.

28 Bjordal JM, Johnson MI, Lopes-Martins RAB, et al. Short-Term efficacy of physical interventions in osteoarthritic knee pain. A systematic review and meta-analysis of randomised placebocontrolled trials. BMC Musculoskelet Disord 2007;8:51.

29 Alfredo PP, Bjordal JM, Dreyer SH, et al. Efficacy of low level laser therapy associated with exercises in knee osteoarthritis: a randomized double-blind study. Clin Rehabil 2012;26:523-33.

30 Fukuda VO, Fukuda TY, Guimarães M, et al. Short-Term efficacy of low-level laser therapy in patients with knee osteoarthritis: a randomized placebo-controlled, double-blind clinical trial. Rev Bras Ortop 2011;46:526-33.
31 Al Rashoud AS, Abboud RJ, Wang W, et al. Efficacy of low-level laser therapy applied at acupuncture points in knee osteoarthritis: a randomised double-blind comparative trial. Physiotherapy 2014;100:242-8.

32 Alghadir A, Omar MTA, Al-Askar AB, et al. Effect of low-level laser therapy in patients with chronic knee osteoarthritis: a single-blinded randomized clinical study. Lasers Med Sci 2014;29:749-55.

33 Gur A, Cosut A, Sarac AJ, et al. Efficacy of different therapy regimes of low-power laser in painful osteoarthritis of the knee: a double-blind and randomized-controlled trial. Lasers Surg Med 2003;33:330-8

34 Tunér J, Hode L. The new laser therapy Handbook: a guide for research scientists, doctors, dentists, veterinarians and other interested parties within the medical field. Grängesberg: Prima Books, 2010.

35 Higgins JPT, Green S. Cochrane Handbook for systematic reviews of interventions, 2011. Available: http://handbook.cochrane.org/ [Accessed 3 Dec 2015].

36 Juhl C, Lund H, Roos EM, et al. A hierarchy of patient-reported outcomes for meta-analysis of knee osteoarthritis trials: empirical evidence from a survey of high impact journals. Arthritis 2012;2012:1-17.

37 Bolognese JA, Schnitzer TJ, Ehrich EW. Response relationship of vas and Likert scales in osteoarthritis efficacy measurement. Osteoarthritis Cartilage 2003;11:499-507.

38 Higgins JPT, Thompson SG, Deeks JJ, et al. Measuring inconsistency in meta-analyses. BMJ 2003;327:557-60.

39 Delkhosh CT, Fatemy E, Ghorbani R, et al. Comparing the immediate and long-term effects of low and high power laser on the symptoms of knee osteoarthritis [Persian]. Journal of mazandaran university of medical sciences 2018;28:69-77.

40 Tascioglu F, Armagan O, Tabak Y, et al. Low power laser treatment in patients with knee osteoarthritis. Swiss Med Wkly 2004;134:254-8

41 Hinman RS, McCrory P, Pirotta M, et al. Acupuncture for chronic knee pain: a randomized clinical trial. JAMA 2014;312:1313-22.

42 Youssef EF, Muaidi QI, Shanb AA. Effect of laser therapy on chronic osteoarthritis of the knee in older subjects. $J$ Lasers Med Sci 2016;7:112-9.

43 Rayegani SM, Bahrami MH, Elyaspour D, et al. Therapeutic effects of low level laser therapy (LLLT) in knee osteoarthritis, compared to therapeutic ultrasound. J Lasers Med Sci 2012;3:71-4.

44 Tubach F, Ravaud P, Baron G, et al. Evaluation of clinically relevant changes in patient reported outcomes in knee and hip osteoarthritis: the minimal clinically important improvement. Ann Rheum Dis 2005;64:29-33.

45 Bellamy N, Hochberg M, Tubach F, et al. Development of multinational definitions of minimal clinically important improvement and patient acceptable symptomatic state in osteoarthritis. Arthritis Care Res 2015;67:972-80.

46 Tubach F, Ravaud P, Martin-Mola E, et al. Minimum clinically important improvement and patient acceptable symptom state in pain and function in rheumatoid arthritis, ankylosing spondylitis, chronic back pain, hand osteoarthritis, and hip and knee osteoarthritis: results from a prospective multina. Arthritis Care Res 2012;64:1699-707.

47 Kheshie AR, Alayat MSM, Ali MME. High-Intensity versus low-level laser therapy in the treatment of patients with knee osteoarthritis: a randomized controlled trial. Lasers Med Sci 2014;29:1371-6.

48 Nambi SG, Kamal W, George J, et al. Radiological and biochemical effects (CTX-II, MMP-3, 8, and 13) of low-level laser therapy (LLLT) in chronic osteoarthritis in Al-Kharj, Saudi Arabia. Lasers Med Sci 2016;32.

49 Lopes-Martins RAB, Marcos RL, Leal-Junior ECP, et al. Low-Level laser therapy and world association for laser therapy dosage recommendations in musculoskeletal disorders and injuries. Photomed Laser Surg 2018;36:457-9.

50 Yurtkuran M, Alp A, Konur S, et al. Laser acupuncture in knee osteoarthritis: a double-blind, randomized controlled study. Photomed Laser Surg 2007;25:14-20.

51 Scott DL, Berry H, Capell H, et al. The long-term effects of non-steroidal anti-inflammatory drugs in osteoarthritis of the knee: a randomized placebo-controlled trial. Rheumatology 2000;39:1095-101.

52 Alfredo PP, Bjordal JM, Junior WS, et al. Long-Term results of a randomized, controlled, double-blind study of low-level laser therapy before exercises in knee osteoarthritis: laser and exercises in knee osteoarthritis. Clin Rehabil 2018:32:173-8.

53 Hegedüs B, Viharos L, Gervain M, et al. The effect of low-level laser in knee osteoarthritis: a double-blind, randomized, placebocontrolled trial. Photomed Laser Surg 2009;27:577-84. 
54 Helianthi DR, Simadibrata C, Srilestari A, et al. Pain reduction after laser acupuncture treatment in geriatric patients with knee osteoarthritis: a randomized controlled trial. Acta Med Indones 2016;48:114-21.

55 Koutenaei FR, Mosallanezhad Z, Naghikhani M, et al. The effect of low level laser therapy on pain and range of motion of patients with knee osteoarthritis. Physical Treatments - Specific Physical Therapy 2017;7:13-18.

56 Mohammed N, Allam H, Elghoroury E, et al. Evaluation of serum beta-endorphin and substance $\mathrm{P}$ in knee osteoarthritis patients treated by laser acupuncture. J Complement Integr Med 2018;15. 